sites are nonoverlapping, thus suggesting that target-membrane Q-SNARE and vesiclemembrane R-SNARE can be accommodated simultaneously. Crucially, overlay of the two Vps33-SNARE crystal structures shows that if the two SNAREs were bound concomitantly, the SNARE helices would line up in parallel to each other, and the zero-layer $\mathrm{R}$ and $\mathrm{Q}$ residues would be aligned perfectly for SNARE-complex assembly (Fig. 2a).

Together, the evidence strongly suggests that a primary role of Vps33 is to orchestrate the precise alignment of cognate SNARE zippers from target and vesicle membranes. This function would support SNARE assembly and membrane fusion, in much the same way that enzymes correctly position substrates and cofactors together for chemical catalysis. If such a catalytic, templating role were translated to the broader SM-protein family, it would help to explain the essentiality of SM proteins in membrane fusion.

The Vps33 R-SNARE-binding site is located on a highly conserved groove of domain $3 \mathrm{a}$ and includes a key interaction with a highly conserved R-SNARE phenylalanine (F201) (Fig. 2a). Indirect evidence has pointed to a potential R-SNARE interaction with domain $3 \mathrm{a}$ and to a role in SNARE assembly and membrane fusion for Munc18a and Sec1 (refs. 7-12). Together, these findings support the notion that concomitant R-SNARE and Q-SNARE binding is a common feature of SM proteins, but this now remains to be firmly established for SM proteins other than Vps33.
In the Munc18a-Syntaxin1a complex, domain 3 a adopts a folded-back conformation that is unfurled in other structures including the Vps33-SNARE assemblies. This means that a conformational change could potentially convert Munc18a from inhibitory (accommodating closed $\mathrm{Q}_{\mathrm{a}}$-SNARE and blocking R-SNARE binding) to activating (binding $\mathrm{Q}_{\mathrm{a}}$-SNARE and R-SNARE simultaneously) (Fig. 2b). The newly identified R-SNAREbinding site on Vps33 allows this hypothesis to be tested in individual systems. Other SM-protein structures now can provide clues to fusion-specific regulatory mechanisms. For example, a loop in yeast Slylp covers the R-SNARE-binding groove, and deletion of the loop results in dominant gain of function, thus suggesting a unique regulatory mechanism for endoplasmic reticulum-Golgi SNARE assembly and membrane fusion ${ }^{13}$.

Of course, many questions still remain. Vps33 is an obligate subunit of a larger tethering complex, and it was crystallized in the presence of HOPS subunit Vps16 as well as the SNARE motifs. Might the Vps16 partner preorganize Vps33 so that it recognizes the open $\mathrm{Q}_{\mathrm{a}}$-SNARE conformation specifically? If so, perhaps SM proteins that are not part of tethering complexes may require external factors to 'open' closed $\mathrm{Q}_{\mathrm{a}}$-SNAREs. Indeed, this is a proposed role for Munc13 in neurotransmission ${ }^{14}$. Moreover, the highly conserved N-peptide interaction is absent in tethering-complex SM proteins including Vps33 (ref. 15). What role does it have in the function of other SM proteins? What happens to the $\mathrm{Q}_{\mathrm{bc}}$ SNAREs, and how do they figure in this templating model? Clearly, the specific and detailed mechanisms by which SM proteins operate will keep researchers occupied for some time.

Nevertheless, there is now an exciting new landscape in which to conduct those studies. The work of Baker et al. ${ }^{2}$ reveals that SM proteins could bind SNAREs from vesicle and target membranes concomitantly, in a manner that would catalyze correct assembly of SNARE complexes. This points to a common function of the enigmatic SM proteins in driving membrane fusion.

\section{COMPETING FINANCIAL INTERESTS}

The authors declare no competing financial interests.

1. Ray, K. J. Biosci. 39, 3-12 (2014).

2. Baker, R.W. et al. Science 349, 1111-1114 (2015).

3. Sutton, R.B., Fasshauer, D., Jahn, R. \& Brunger, A.T. Nature 395, 347-353 (1998).

4. Misura, K.M., Scheller, R.H. \& Weis, W.I. Nature 404 , 355-362 (2000).

5. Burkhardt, P., Hattendorf, D.A., Weis, W.I. \& Fasshauer, D. EMBO J. 27, 923-933 (2008).

6. Burkhardt, P. et al. Proc. Natl. Acad. Sci. USA 108 15264-15269 (2011).

7. Hu, S.H. et al. Proc. Natl. Acad. Sci. USA 108 1040-1045 (2011).

8. Parisotto, D. et al. J. Biol. Chem. 289, 9639-9650 (2014).

9. Martin, S. et al. J. Cell Sci. 126, 2353-2360 (2013).

10. Han, G.A., Bin, N.R., Kang, S.Y., Han, L. \& Sugita, S. J. Cell Sci. 126, 2361-2371 (2013).

11. Boyd, A. et al. Genetics 180, 165-178 (2008).

12. Xu, Y., Su, L. \& Rizo, J. Biochemistry 49, 1568-1576 (2010).

13. Li, Y., Schmitt, H.D., Gallwitz, D. \& Peng, R.W. FEBS Lett. 581, 5698-5702 (2007).

14. Yang, X. et al. Nat. Struct. Mol. Biol. 22, 547-554 (2015).

15. Hu, S.H., Latham, C.F., Gee, C.L., James, D.E. \& Martin, J.L. Proc. Natl. Acad. Sci. USA 104 8773-8778 (2007).

\title{
Reversible aggregation after heat shock
}

In eukaryotic cells, heat stress triggers aggregation of proteins and RNA into heat-shock granules (HSGs). However, the formation and composition of HSGs remain poorly understood. Drummond and co-workers now provide a comprehensive proteomic analysis of heattriggered aggregation and subsequent disaggregation in budding yeast (Cell 162, 1286-1298, 2015).

The authors monitored aggregation of proteins into highmolecular-weight particles by ultracentrifugation and fractionation into supernatant and pellet. Stable-isotope labeling and mass spectrometry enabled estimation of the proportion of each protein in the supernatant before and after heat shock. After a shift from $30{ }^{\circ} \mathrm{C}$ to $46{ }^{\circ} \mathrm{C}, 177$ proteins moved from the supernatant to the pellet, and only four moved from the pellet to the supernatant. Molecular chaperones such as Hsp104, which colocalize with HSGs, remained largely soluble, thus suggesting a biochemical distinction between aggregation and recruitment to aggregates. Interestingly, individual proteins aggregated in distinct subcellular compartments. For example, the nuclear poly(A) RNAbinding protein Gbp2 formed subnuclear granules during heat shock (left), whereas the HSG marker Pab1 formed cytosolic foci (left middle).

Next, the authors asked how aggregation affects protein function. AME, a heterotrimeric aminoacyl-tRNA synthetase complex, aggregated upon heat shock but, surprisingly, it retained substantial activity and fidelity in vitro. The authors suggest that AME aggregation could focus its activity in the cytosol, where it is needed during stress. Finally, the authors globally profiled disaggregation after recovery. Unexpectedly, they did not observe degradation of disaggregated proteins; instead, proteins were restored to solubility without degradation.

These findings should spur reinterpretation of heat stress-induced events: aggregates of mature proteins probably represent an adaptive cellular strategy rather than simply being misfolded proteins destined for degradation. 